\title{
Colitis cystica profunda mimicking mucinous adenocarcinoma of the rectum diagnosed by endoscopic submucosal dissection
}

A 48-year-old man presented to our hospital with complaints of fresh rectal bleeding. He was intellectually disabled and often inserted his fingers into the anus after defecation. Laboratory investigations demonstrated lowered hemoglobin level $(9.3 \mathrm{~g} / \mathrm{dL}$; normal range $13.5-$ $17.5 \mathrm{~g} / \mathrm{dL}$ ). Colonoscopy showed a polypoid submucosal tumor-like elevated lesion with redness and rough surface, along with erosion in the lower rectum ( Fig.1a,b). Endoscopic ultrasonography (EUS) showed hypoechoic and several anechoic cystic lesions in the submucosa, without invasion of the muscularis propria ( Fig. $1 \mathrm{c}$ ). Magnetic resonance imaging (MRI) showed peripheral contrast-enhanced cystic lesions in the lower rectum. Regional lymphadenitis in the lower rectum was not seen on MRI ( Fig.2). Boring biopsy was suggestive of mucinous adenocarcinoma.

After obtaining informed consent, we performed endoscopic submucosal dissection (ESD) for complete en bloc resection to confirm the preoperative diagnosis ( Fig. 1 d, > Fig. 3 a, > Video 1). Histological examination revealed colitis cystica profunda (CCP) with no evidence

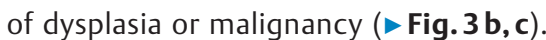
The patient progressed well after ESD and was discharged 7 days postoperatively. The patient was treated conservatively to improve bowel habits.

CCP is a rare benign disease characterized by mucin-filled cysts beneath the muscularis mucosa [1]. Knowledge of this pathological entity is important as it can mimic a well-differentiated adenocarcinoma of the rectum and therefore could lead to unnecessary surgical resection [2]. EUS, computed tomography, and MRI are useful for diagnosis of CCP $[3,4]$; however, the definitive diagnosis is based on endoscopic biopsies, which should be large and deep enough to include the submucosal layer, or resected surgical
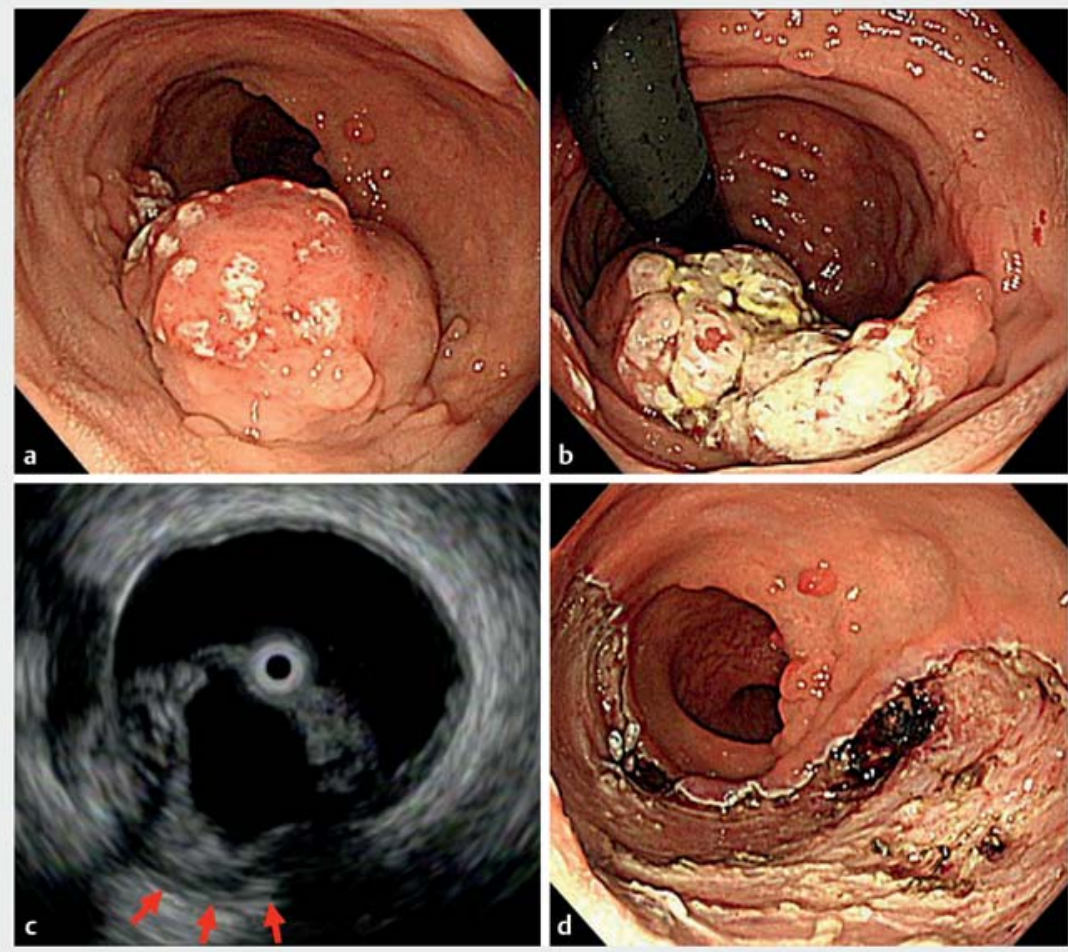

Fig. 1 Examination and imaging of the lesion. a, b Colonoscopy showed a polypoid submucosal tumor-like elevated lesion with redness and rough surface, and erosion in the lower rectum, approximately $7 \mathrm{~cm}$ in size. c Endoscopic ultrasonography showed hypoechoic and several anechoic cystic lesions located in the submucosa without invasion of the muscularis propria (red arrows). d The mucosa immediately after endoscopic submucosal dissection.
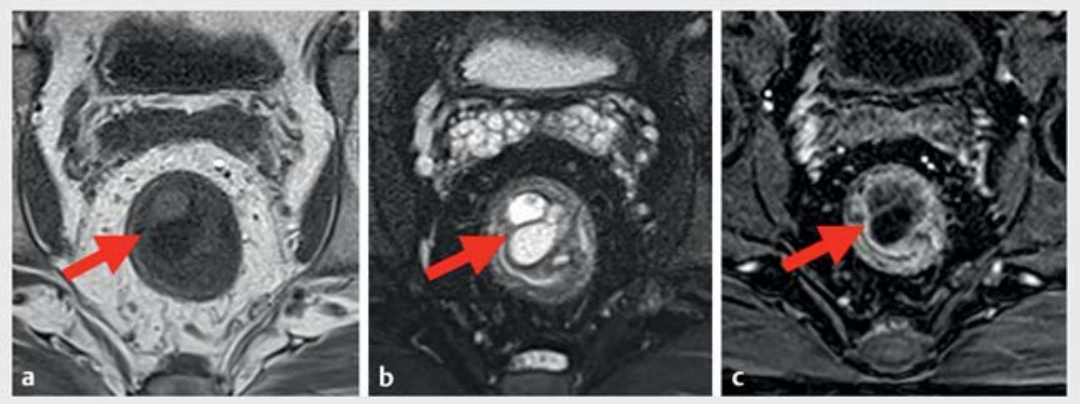

Fig. 2 Abdominal magnetic resonance imaging (MRI). a, b Homogenous and variable hypointense nodules on T1-weighted MRI (a) and hyperintense nodules on T2-weighted fat-suppressed MRI (b) showed no infiltrative signs and regional swollen lymph nodes in the lower rectum (red arrow). c Contrast-enhanced images on T2-weighted fat-suppressed MRI showed peripheral enhancement (red arrow). 

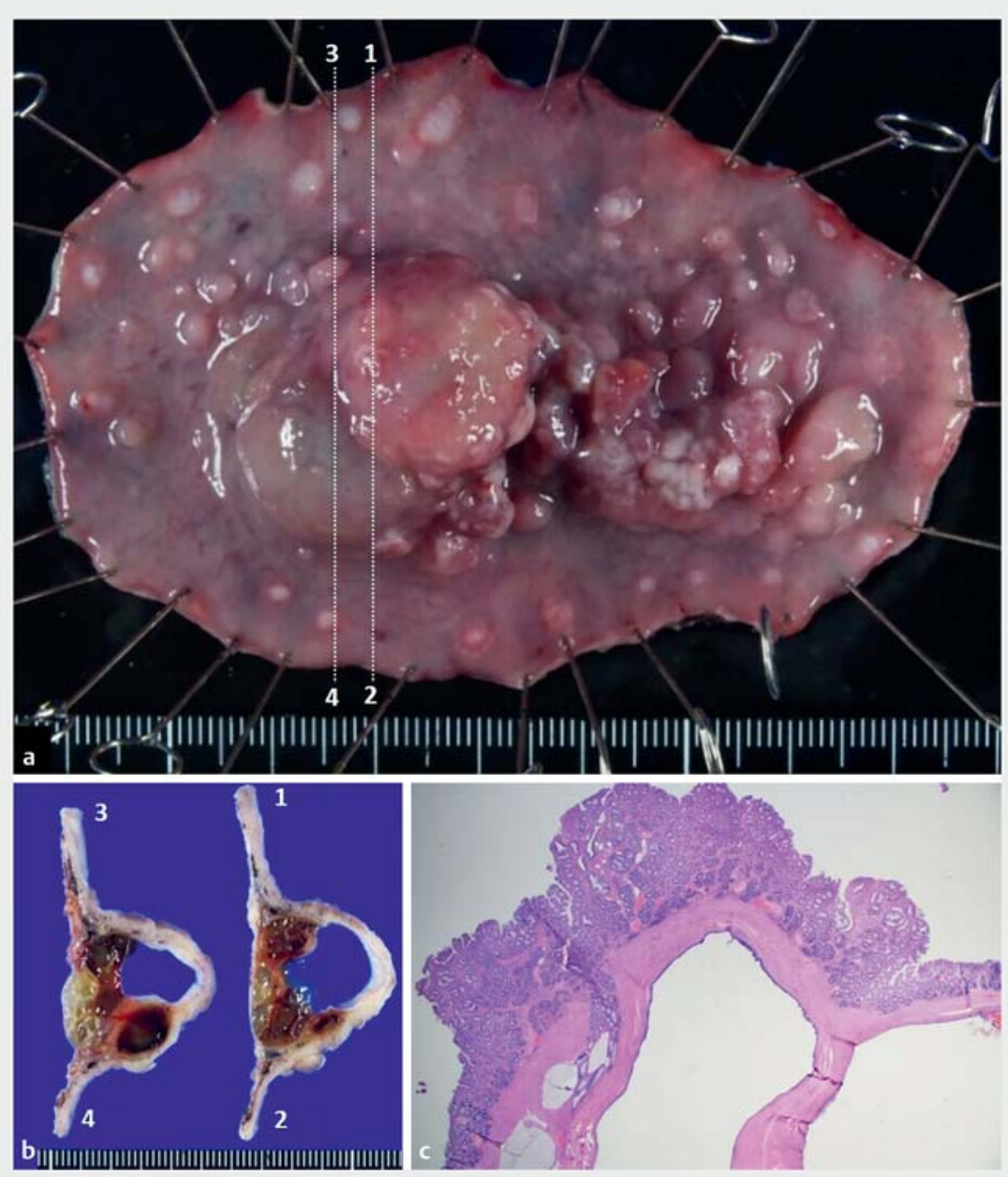

- Fig. 3 The resected specimen. a Photograph of the resected specimen, approximately $9 \times 6 \mathrm{~cm}$ in size. b Macroscopic view of the resected specimen, showing several cystic lesions containing mucin. $\mathbf{c}$ Histological images of the resected specimen stained with hematoxylin and eosin, showing colitis cystica profunda without findings of dysplasia or malignancy $(\times 12.5)$.
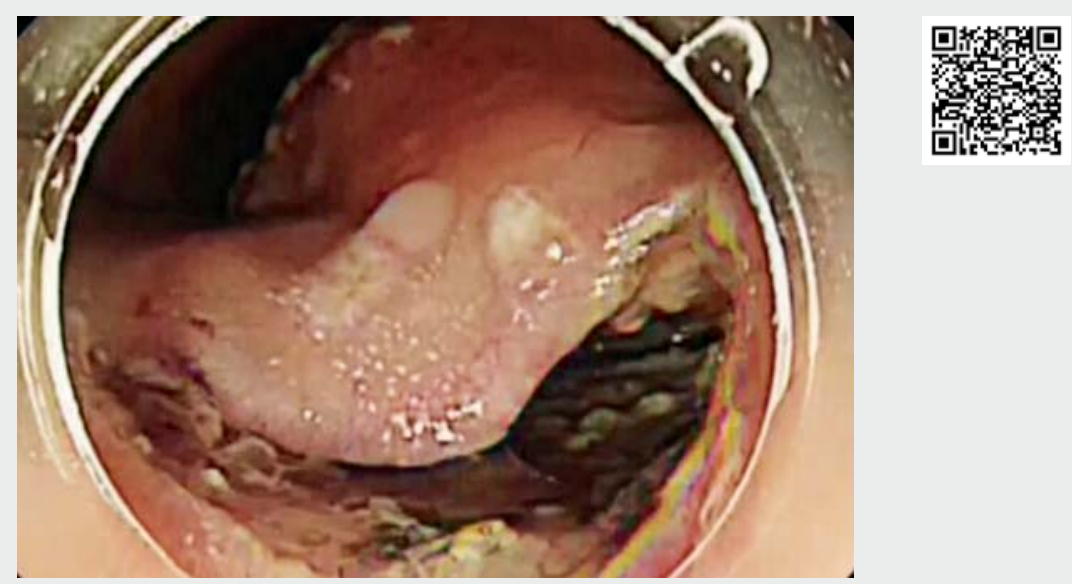

$\checkmark$ Video 1 Endoscopic submucosal dissection of colitis cystica profunda in the lower rectum along with resection of the tumor using the pocket-creation and clip-with-line methods. specimens, as small endoscopic biopsies are frequently unrevealing [5]. ESD may provide an endoscopic technique for diagnosis and treatment of CCP.

Endoscopy_UCTN_Code_CCL_1AD_2AC

\section{Competing interests}

The authors declare that they have no conflict of interest.

\section{The authors}

Takashi Abe' ${ }^{1}$, Marina Hamamoto', Takayuki Nagai ${ }^{1}$, Takehiko Nariyasu', Masato

Hanzawa ${ }^{1}$, Yasuhisa Hiroshima ${ }^{1}$, Kazunari Murakami $^{2}$

1 Department of Gastroenterology, Oita Kouseiren Tsurumi Hospital, Beppu, Japan

2 Department of Gastroenterology, Faculty of Medicine, Oita University, Yufu, Japan

\section{Corresponding author}

\section{Takashi Abe, MD, PhD}

Department of Gastroenterology, Oita Kouseiren Tsurumi Hospital, Tsurumi 4333, Beppu City, Oita 874-8585, Japan

Fax: +81-977-237884

takashi0315@oita-u.ac.jp 


\section{References}

[1] Kornprat P, Langner C, Pfeifer J et al. Colitis cystica profunda associated with rectal prolapse: report of a case. Int J Colorectal Dis 2007; 22: 1555-1556

[2] Kayaçetin E, Kayaçetin S. Colitis cystica profunda simulating rectal carcinoma. Acta Chi Belg 2005; 105: 306-308

[3] Valenzuela M, Martín-Ruiz JL, Alvarez-Cienfuegos E et al. Colitis cystica profunda: imaging diagnosis and conservative treatment: report of two cases. Dis Colon Rectum 1996; 39: $587-590$

[4] Rumi N, Cilla S, De Ninno M et al. Colitis cystica profunda of the rectum with adenomatous dysplastic features: radiologic- pathologic correlation. Radiol Case Rep 2019; 14: 740-745

[5] Spicakova K, Pueyo BA, de la Piscina PR et al. Colitis cystica profunda: a report of 2 cases with a 15-year follow-up. Gastroenterol Hepatol 2017; 40: 406-408

\section{Bibliography}

Endoscopy 2021; 53: E157-E159

DOI 10.1055/a-1224-3347

ISSN 0013-726X

published online 20.8.2020

(c) 2020. Thieme. All rights reserved.

Georg Thieme Verlag KG, Rüdigerstraße 14,

70469 Stuttgart, Germany

\section{ENDOSCOPY E-VIDEOS}

https:/|eref.thieme.de/e-videos

口回 Endoscopy E-Videos is a free access online section, reporting 田: on interesting cases and new techniques in gastroenterological endoscopy. All papers include a high quality video and all contributions are freely accessible online.

This section has its own submission website at

https://mc.manuscriptcentral.com/e-videos 